\title{
SENSITIVITY ANALYSIS FOR A SYSTEM OF GENERALIZED NONLINEAR MIXED QUASI-VARIATIONAL INCLUSIONS WITH $(A, \eta)$-ACCRETIVE MAPPINGS IN BANACH SPACES
}

\author{
JaE UG JeOng and Soo Hwan KIm
}

\begin{abstract}
In this paper, we study the behavior and sensitivity analysis of the solution set for a new system of parametric generalized nonlinear mixed quasi-variational inclusions with $(A, \eta)$-accretive mappings in $q$ uniformly smooth Banach spaces. The present results improve and extend many known results in the literature.
\end{abstract}

\section{Introduction}

Sensitivity analysis of solutions of variational inequalities with single-valued mappings has been studied by many authors via quite different techniques.

By using the projection method, Dafermos [2], Yen [9], Mukherjee and Verma [6], Noor [7], and Pan [8] studied the sensitivity analysis of solutions of some variational inequalities with single-valued mappings in finite-dimensional spaces or Hilbert spaces.

In 2004, using the concept and technique of resolvent operators, Agawal et. al [1] introduced and studied the behavior and sensitivity analysis of the solution set for a system of parametric variational inclusions in a Hilbert space $H$, which is called the system of parametric generalized nonlinear mixed quasivariational inclusion problem:

For a given two nonempty open subsets $\Omega$ and $\Lambda$ of $H$ in which the parameters $\omega$ and $\lambda$ take values, two maximal monotone mappings $M: H \times \Omega \rightarrow 2^{H}$ and $N: H \times \Lambda \rightarrow 2^{H}$, nonlinear single-valued mappings $A, S: H \times \Omega \rightarrow H$ and $B, T: H \times \Lambda \rightarrow H$, find $(x, y) \in H \times H$ such that

$$
\begin{aligned}
& 0 \in x-y+\rho(A(y, \omega)+S(y, \omega))+\rho M(x, \omega), \\
& 0 \in y-x+\gamma(B(x, \lambda)+T(x, \lambda))+\gamma N(y, \lambda),
\end{aligned}
$$

where $\rho, \lambda>0$ are two constants.

Received September 19, 2008; Revised December 5, 2008.

2000 Mathematics Subject Classification. 49J40, 90C33.

Key words and phrases. quasi-variational inclusion, sensitivity analysis, resolvent operator, $(A, \eta)$-accretive mapping. 
In this paper, we study the behavior and sensitivity analysis of the solution set for a system of parametric generalized nonlinear mixed quasi-variational inclusions with $(A, \eta)$-accretive mappings in $q$-uniformly smooth Banach spaces. The present results improve and extend many known results in the literature.

\section{Preliminaries}

Let $E$ be a real Banach space with dual space $E^{*},\langle\cdot, \cdot\rangle$ be the dual pair between $E$ and $E^{*}$ and $2^{E}$ denote the family of all the nonempty subsets of $E$. The generalized duality mapping $J_{q}: E \rightarrow 2^{E^{*}}$ is defined by

$$
J_{q}(x)=\left\{f \in E^{*} \mid\left\langle x, f^{*}\right\rangle=\|x\|^{q} \text { and }\left\|f^{*}\right\|=\|x\|^{q-1}\right\}, \forall x \in E,
$$

where $q>1$ is a constant. In particular, $J_{2}$ is the usual normalized duality mapping. It is known that, in general, $J_{q}(x)=\|x\|^{q-2} J_{2}(x)$ for all $x \neq 0$ and $J_{q}$ is single-valued if $E^{*}$ is strictly convex. If $E=H$ is a Hilbert space, then $J_{2}$ becomes the identity mapping of $H$. by

The modulus of smoothness of $E$ is the function $\rho_{E}:[0, \infty) \rightarrow[0, \infty)$ defined

$$
\rho_{E}(t)=\sup \left\{\frac{1}{2}(\|x+y\|+\|x-y\|)-1:\|x\| \leq 1,\|y\| \leq t\right\} .
$$

A Banach space $E$ is called uniformly smooth if $\lim _{t \rightarrow 0} \frac{\rho_{E}(t)}{t}=0$. $E$ is called $q$-uniformly smooth if there exists a constant $c>0$ such that $\rho_{E}(t) \leq c t^{q}$, $q>1$. Note that $J_{q}$ is single-valued if $E$ is uniformly smooth.

We consider now a system of parametric generalized nonlinear mixed quasivariational inclusions with $(A, \eta)$-accretive mappings in $q$-uniformly smooth Banach spaces. To this end, let $\Omega$ and $\Lambda$ be two nonempty open subsets of $E$ in which the parameters $\omega$ and $\lambda$ take values, $S, T: E \times \Omega \rightarrow E$ and $U, V$ : $E \times \Lambda \rightarrow E$ be nonlinear single-valued mappings. Let $M: E \times \Omega \rightarrow 2^{E}$ and $N: E \times \Lambda \rightarrow 2^{E}$ be set-valued mappings such that for each given $(\omega, \lambda) \in \Omega \times \Lambda$, $M(\cdot, \omega)$ and $N(\cdot, \lambda): E \rightarrow 2^{E}$ are $(A, \eta)$-accretive mappings. For each fixed $(\omega, \lambda) \in \Omega \times \Lambda$, the system of parametric generalized nonlinear mixed quasivariational inclusions with $(A, \eta)$-accretive mappings in $q$-uniformly smooth Banach spaces consist of finding $(x, y) \in E \times E$ such that

$$
\begin{aligned}
& 0 \in A(x)-y+\rho(S(y, \omega)+T(y, \omega))+\rho M(x, \omega), \\
& 0 \in A(y)-x+\gamma(U(x, \lambda)+V(x, \lambda))+\gamma N(y, \lambda),
\end{aligned}
$$

where $\rho>0$ and $\gamma>0$ are two constants.

We now discuss some special cases.

Case I. Let $E=H$ be a Hilbert space, $A=I$, the identity mappings, $\Omega$ and $\Lambda$ be two nonempty open subsets of $H$ in which the parameters $\omega$ and $\lambda$ takes values. Let $\phi_{1}: H \times \Omega \rightarrow R \cup\{+\infty\}$ and $\phi_{2}: H \times \Lambda \rightarrow R \cup\{+\infty\}$ be functionals such that for $(x, \omega) \in H \times \Omega$ and $(y, \lambda) \in H \times \Lambda, \partial \phi_{1}(\cdot, \omega)$ and $\partial \phi_{2}(\cdot, \lambda)$ denote the subdifferential of proper convex lower semicontinuous functions $\phi_{1}$ and $\phi_{2}$, respectively. Let $M(\cdot, \omega)=\partial \phi_{1}(\cdot, \omega)$ and $N(\cdot, \lambda)=\partial \phi_{2}(\cdot, \lambda)$ for all 
$(\omega, \lambda) \in \Omega \times \Lambda$. Then problem (2.1) is equivalent to finding $\left(x^{*}, y^{*}\right) \in H \times H$ such that

$$
\begin{aligned}
& \left\langle\rho\left(S\left(y^{*}, \omega\right)+T\left(y^{*}, \omega\right)\right)+x^{*}-y^{*}, x-x^{*}\right\rangle \geq \rho \phi_{1}\left(x^{*}, \omega\right)-\rho \phi_{1}(x, \omega), \\
& \left\langle\gamma\left(U\left(x^{*}, \lambda\right)+V\left(x^{*}, \lambda\right)\right)+y^{*}-x^{*}, x-y^{*}\right\rangle \geq \gamma \phi_{2}\left(y^{*}, \lambda\right)-\gamma \phi_{2}(x, \lambda)
\end{aligned}
$$

for all $x \in H$, which is called the system of parametric generalized nonlinear mixed variational inequalities in Hilbert spaces [1].

Case II. Let $K$ be a nonempty closed convex subset of $H$ and $\phi_{i}=I_{K}$ $(i=1,2)$ are the indicator functions of $K$. Then problem $(2.2)$ reduces to the problem of finding $\left(x^{*}, y^{*}\right) \in K \times K$ such that

$$
\begin{aligned}
& \left\langle\rho\left(S\left(y^{*}, \omega\right)+T\left(y^{*}, \omega\right)\right)+x^{*}-y^{*}, x-x^{*}\right\rangle \geq 0, \\
& \left\langle\gamma\left(U\left(x^{*}, \lambda\right)+V\left(x^{*}, \lambda\right)\right)+y^{*}-x^{*}, x-y^{*}\right\rangle \geq 0
\end{aligned}
$$

for all $x \in K$, which is called the system of parametric generalized nonlinear quasi-variational inequalities in Hilbert spaces [1].

Definition 2.1. Let $A: E \rightarrow E, \eta: E \times E \rightarrow E$ be two single-valued mappings. Then a multivalued mapping $M: E \rightarrow 2^{E}$ is said to be

(i) accretive if

$$
\left\langle u-v, J_{q}(x-y)\right\rangle \geq 0, \quad \forall x, y \in E, u \in M(x), v \in M(y)
$$

(ii) $m$-relaxed $\eta$-accretive if there exists a constant $m>0$ such that

$$
\left\langle u-v, J_{q}(\eta(x, y))\right\rangle \geq-m\|x-y\|^{q}, \quad \forall x, y \in E, u \in M(x), v \in M(y) ;
$$

(iii) $(A, \eta)$-accretive if $M$ is $m$-relaxed $\eta$-accretive and $(A+\rho M)(E)=E$ for every $\rho>0$.

Definition 2.2. A mapping $S: E \times \Omega \rightarrow E$ is said to be

(i) $\delta$-strongly accretive with respect to the first argument, $\delta \in(0,1)$, if

$$
\left\langle S(x, \omega)-S(y, \omega), J_{q}(x-y)\right\rangle \geq \delta\|x-y\|^{q}, \quad \forall x, y \in E ;
$$

(ii) $\lambda_{S}$-Lipschitz continuous with respect to the first argument if there exists a constant $\lambda_{S}>0$ such that

$$
\|S(x, \omega)-S(y, \omega)\| \leq \lambda_{S}\|x-y\|, \quad \forall(x, y, \omega) \in E \times E \times \Omega .
$$

Definition 2.3. A single-valued mapping $A: E \rightarrow E$ is said to be

(i) $\eta$-accretive if

$$
\left\langle A(x)-A(y), J_{q}(\eta(x, y))\right\rangle \geq 0, \quad \forall x, y \in E ;
$$

(ii) strictly $\eta$-accretive if $A$ is $\eta$-accretive and equality holds if and only if $x=y$.

(iii) $\gamma$-strongly $\eta$-accretive if there exists a constant $\gamma>0$ such that

$$
\left\langle A(x)-A(y), J_{q}(\eta(x, y))\right\rangle \geq \gamma\|x-y\|^{q}, \quad \forall x, y \in E .
$$


If $A: E \rightarrow E$ is a strictly $\eta$-accretive mapping and $M: E \rightarrow 2^{E}$ is an $(A, \eta)$ accretive mapping, then for a constant $\rho>0$, the resolvent operator associated with $A$ and $M$ is defined by

$$
R_{M, \rho}^{A, \eta}(u)=(A+\rho M)^{-1}(u), \quad \forall u \in E .
$$

It is well known that $R_{M, \rho}^{A, \eta}$ is a single-valued mapping [5].

Remark 2.1. Since $M$ is an $(A, \eta)$-accretive mapping with respect to the first argument, for any fixed $\omega \in \Omega$, we define

$$
R_{M(\cdot, \omega), \rho}^{A, \eta}(u)=(A+\rho M(\cdot, \omega))^{-1}(u), \quad \forall u \in D(M),
$$

which is called the parametric resolvent operator associated with $A$ and $M(\cdot, \omega)$.

Remark 2.2. Resolvent operators associated with $(A, \eta)$-accretive mappings include as special cases the corresponding resolvent operators associated with $(H, \eta)$-accretive operators [8], $(H, \eta)$-monotone operators [10], $H$-accretive operators [4], $H$-monotone operators [3], $A$-monotone operators [11], the classical $m$-accretive and maximal monotone operators [14].

Now we need some lemmas which will be used in the proofs for the main results in the next section.

Lemma 2.1 ([12]). Let $E$ be a real uniformly smooth Banach space. Then $E$ is $q$-uniformly smooth if and only if there exists a constant $c_{q}>0$ such that for all $x, y \in E$

$$
\|x+y\|^{q} \leq\|x\|^{q}+q\left\langle y, J_{q}(x)\right\rangle+c_{q}\|y\|^{q} .
$$

Lemma $2.2([5])$. Let $E$ be a q-uniformly smooth Banach space, $\eta: E \times E \rightarrow E$ be $\tau$-Lipschitz continuous, $A: E \rightarrow E$ be r-strongly $\eta$-accretive mapping and $M: E \rightarrow 2^{E}$ be an $(A, \eta)$-accretive mapping. Then the resolvent operator $R_{M(\cdot, \omega), \rho}^{A, \eta}: E \rightarrow E$ is $\frac{\tau^{q-1}}{r-\rho m}-$ Lipschitz continuous, i.e.,

$$
\left\|R_{M(\cdot, \omega), \rho}^{A, \eta}(u)-R_{M(\cdot, \omega), \rho}^{A, \eta}(v)\right\| \leq \frac{\tau^{q-1}}{r-\rho m}\|u-v\|, \quad \forall u, v \in E,
$$

where $\rho \in\left(0, \frac{r}{m}\right)$ is a constant.

\section{Sensitivity analysis of solution set}

Throughout the rest of this paper, we always assume that $E$ is a real $q$ uniformly smooth Banach space. First of all, we prove the following lemma.

Lemma 3.1. For all fixed $(\omega, \lambda) \in \Omega \times \Lambda,(\bar{x}(\omega, \lambda), \bar{y}(\omega, \lambda))$ is a solution of the system of parametric generalized nonlinear quasi-variational inclusions with $(A, \eta)$-accretive mapping in q-uniformly smooth Banach space (2.1) if and only if for some given $\rho, \gamma>0$, the mapping $F: E \times \Omega \times \Lambda \rightarrow E$ defined by 


$$
\begin{aligned}
F(x, \omega, y)= & R_{M(\cdot, \omega), \rho}^{A, \eta}\left[R_{N(\cdot, \lambda), \gamma}^{A, \eta}(x-\gamma(U+V)(x, \lambda))\right. \\
& \left.-\rho(S+T)\left(R_{N(\cdot, \lambda), \gamma}^{A, \eta}(x-\gamma(U+V)(x, \lambda)), \omega\right)\right]
\end{aligned}
$$

has a fixed point $\bar{x}$.

Proof. For each fixed $(\omega, \lambda) \in \Omega \times \Lambda$, let $(\bar{x}(\omega, \lambda), \bar{y}(\omega, \lambda))$ be a solution of problem (2.1). Then for given $\rho, \gamma>0$,

$$
\begin{aligned}
& 0 \in A(\bar{x})-\bar{y}+\rho(S(\bar{y}, \omega)+T(\bar{y}, \omega))+\rho M(\bar{x}, \omega), \\
& 0 \in A(\bar{y})-\bar{x}+\gamma(U(\bar{x}, \lambda)+V(\bar{x}, \lambda))+\gamma N(\bar{y}, \lambda),
\end{aligned}
$$

which implies that

$$
\begin{aligned}
& \bar{y}-\rho(S+T)(\bar{y}, \omega) \in[A+\rho M(\cdot, \omega)](\bar{x}), \\
& \bar{x}-\gamma(U+V)(\bar{x}, \lambda) \in[A+\gamma N(\cdot, \lambda)](\bar{y}) .
\end{aligned}
$$

Thus, we obtain

$$
\begin{aligned}
& (A+\rho M(\cdot, \omega))^{-1}[\bar{y}-\rho(S+T)(\bar{y}, \omega)]=\bar{x}, \\
& (A+\gamma N(\cdot, \lambda))^{-1}[\bar{x}-\gamma(U+V)(\bar{x}, \lambda)]=\bar{y},
\end{aligned}
$$

i.e.,

Hence, we have

$$
\begin{aligned}
& R_{M(\cdot, \omega), \rho}^{A, \eta}[\bar{y}-\rho(S+T)(\bar{y}, \omega)]=\bar{x} \\
& R_{N(\cdot, \lambda), \gamma}^{A, \eta}[\bar{x}-\gamma(U+V)(\bar{x}, \lambda)]=\bar{y} .
\end{aligned}
$$

$$
\begin{aligned}
\bar{x}= & R_{M(\cdot, \omega), \rho}^{A, \eta}\left[R_{N(\cdot, \lambda), \gamma}^{A, \eta}(\bar{x}-\gamma(U+V)(\bar{x}, \lambda))\right. \\
& \left.-\rho(S+T)\left(R_{N(\cdot, \lambda), \gamma}^{A, \eta}(\bar{x}-\gamma(U+V)(\bar{x}, \lambda)), \omega\right)\right] \\
= & F(\bar{x}, \omega, \lambda) .
\end{aligned}
$$

This means that $\bar{x}$ is a fixed point of $F(x, \omega, \lambda)$.

Now, for any fixed point $(\omega, \lambda) \in(\Omega \times \Lambda)$, let $\bar{x}$ be a fixed point of $F(x, \omega, \lambda)$. By the definition of $F$,

$$
\begin{aligned}
\bar{x}= & F(\bar{x}, \omega, \lambda) \\
= & R_{M(\cdot, \omega), \rho}^{A, \eta}\left[R_{N(\cdot, \lambda), \gamma}^{A, \eta}(\bar{x}-\gamma(U+V)(\bar{x}, \lambda))\right. \\
& \left.-\rho(S+T)\left(R_{N(\cdot, \lambda), \gamma}^{A, \eta}(\bar{x}-\gamma(U+V)(\bar{x}, \lambda)), \omega\right)\right] .
\end{aligned}
$$

Let

Then we have

$$
\bar{y}=R_{N(\cdot, \lambda), \gamma}^{A, \eta}(\bar{x}-\gamma(U+V)(\bar{x}, \lambda)) .
$$

$$
\bar{x}=R_{M(\cdot, \omega), \rho}^{A, \eta}[\bar{y}-\rho(S+T)(\bar{y}, w)] .
$$

By the definitions of $R_{M(\cdot, \omega), \rho}^{A, \eta}$ and $R_{N(\cdot, \lambda), \gamma}^{A, \eta}$, we get

$$
\bar{x}=(A+\rho M(\cdot, \omega))^{-1}[\bar{y}-\rho(S+T)(\bar{y}, \omega)],
$$




$$
\bar{y}=(A+\gamma N(\cdot, \lambda))^{-1}[\bar{x}-\gamma(U+T)(\bar{x}, \lambda)],
$$

which implies that

$$
\begin{aligned}
& \bar{y}-\rho(S+T)(\bar{y}, \omega) \in[A+\rho M(\cdot, \omega)](\bar{x}), \\
& \bar{x}-\gamma(U+V)(\bar{x}, \lambda) \in[A+\gamma N(\cdot, \lambda)](\bar{y}) .
\end{aligned}
$$

Hence

$$
\begin{aligned}
& 0 \in A(\bar{x})-\bar{y}+\rho(S(\bar{y}, \omega)+T(\bar{y}, \omega))+\rho M(\bar{x}, \omega), \\
& 0 \in A(\bar{y})-\bar{x}+\gamma(U(\bar{x}, \lambda)+V(\bar{x}, \lambda))+\gamma N(\bar{y}, \lambda) .
\end{aligned}
$$

This completes the proof.

Theorem 3.1. Let $A: E \rightarrow E, S, T: E \times \Omega \rightarrow E, U, V: E \times \Lambda \rightarrow E$ be five mappings and $M: E \times \Omega \rightarrow 2^{E}, N: E \times \Lambda \rightarrow 2^{E}$ be two set-valued mappings satisfying the following conditions:

(i) A is r-strongly $\eta$-accertive mapping,

(ii) $S$ is $\lambda_{S}$-Lipschitz continuous with respect to the first argument,

(iii) $T$ is $\delta$-strongly accretive and $\lambda_{T}$-Lipschitz continuous with respect to the first argument,

(iv) $U$ is $\lambda_{U}$-Lipschitz continuous with respect to the first argument,

(v) $V$ is $\alpha$-strongly accretive and $\lambda_{V}$-Lipschitz continuous with respect to the first argument,

(vi) $M$ and $N$ are $(A, \eta)$-accretive with respect to the first argument.

Suppose that there exist $\rho>0$ and $\gamma>0$ such that

$$
\begin{gathered}
1-\alpha q \gamma+c_{q} \gamma^{q} \lambda_{V}^{q}<\left(\frac{\gamma-\rho m}{\tau^{q-1}}-\gamma \lambda_{U}\right)^{q}, \\
1-q \rho \delta+c_{q} \rho^{q} \lambda_{T}^{q}<\left(\frac{\gamma-\lambda m}{\tau^{q-1}}-\rho \lambda_{S}\right)^{q} .
\end{gathered}
$$

Then

(1) the mapping $F: E \times \Omega \times \Lambda \rightarrow E$ defined by (3.1) is a uniform $\theta$-contractive mapping with respect to $(\omega, \lambda) \in \Omega \times \Lambda$.

(2) for each $(\omega, \lambda) \in \Omega \times \Lambda$, the system of parametric generalized nonlinear mixed quasi-variational inclusions with $(A, \eta)$-accretive mappings in $q$ uniformly smooth Banach spaces (2.1) has a nonempty solution set $S(\omega, \lambda)$ and $S(\omega \lambda)$ is a closed subset of $E$.

Proof. (1) By the definition of $F$, for any $x, y \in E$, we have

$$
\begin{aligned}
& \|F(x, \omega, \lambda)-F(y, \omega, \lambda)\| \\
& =\| R_{M(\cdot, \omega), \rho}^{A, \eta}\left[R_{N(\cdot, \lambda), \gamma}^{A, \eta}(x-\gamma(U+V)(x, \lambda))\right. \\
& \left.\quad-\rho(S+T)\left(R_{N(\cdot, \lambda), \gamma}^{A, \eta}(x-\gamma(U+V)(x, \lambda)), \omega\right)\right] \\
& \quad-\quad R_{M(\cdot, \omega), \rho}^{A, \eta}\left[R_{N(\cdot, \lambda), \gamma}^{A, \eta}(y-\gamma(U+V)(y, \lambda))\right.
\end{aligned}
$$




$$
\begin{aligned}
& \left.-\rho(S+T)\left(R_{N(\cdot, \lambda), \gamma}^{A, \eta}(y-\gamma(U+V)(y, \lambda)), \omega\right)\right] \| \\
& \leq \frac{\tau^{q-1}}{r-\rho m} \| R_{N(\cdot, \lambda), \gamma}^{A, \eta}(x-\gamma(U+V)(x, \lambda)) \\
& \quad-\rho(S+T)\left(R_{N(\cdot, \lambda), \gamma}^{A, \eta}(x-\gamma(U+V)(x, \lambda)), \omega\right) \\
& \quad-\left(R_{N(\cdot, \lambda), \gamma}^{A, \eta}(y-\gamma(U+V)(y, \lambda))\right. \\
& \left.\quad-\rho(S+T)\left(R_{N(\cdot, \lambda), \gamma}^{A, \eta}(y-\gamma(U+V)(y, \lambda)), \omega\right)\right) \| \\
& \leq \frac{\tau^{q-1}}{r-\rho m} \| R_{N(\cdot, \lambda), \gamma}^{A, \eta}(x-\gamma(U+V)(x, \lambda))-R_{N(\cdot, \lambda), \gamma}^{A, \eta}(y-\gamma(U+V)(y, \lambda)) \\
& -\rho\left[T\left(R_{N(\cdot, \lambda), \gamma}^{A, \eta}(x-\gamma(U+V)(x, \lambda)), \omega\right)\right. \\
& \left.\quad-T\left(R_{N(\cdot, \lambda), \gamma}^{A, \eta}(y-\gamma(U+V)(y, \lambda)), \omega\right)\right] \| \\
& +\frac{\tau^{q-1}}{r-\rho m} \rho \| S\left(R_{N(\cdot, \lambda), \gamma}^{A, \eta}(x-\gamma(U+V)(x, \lambda)), \omega\right) \\
& \quad-S\left(R_{N(\cdot, \lambda), \gamma}^{A, \eta}(y-\gamma(U+V)(y, \lambda)), \omega\right) \| .
\end{aligned}
$$

From Lemma 2.1, the $\delta$-strong accretivity and $\lambda_{T}$-Lipschitz continuity of $T$ it follows that

$$
\begin{aligned}
& \| R_{N(\cdot, \lambda), \gamma}^{A, \eta}(x-\gamma(U+V)(x, \lambda))-R_{N(\cdot, \lambda), \gamma}^{A, \eta}(y-\gamma(U+V)(y, \lambda)) \\
& -\rho\left\{T\left(R_{N(\cdot, \lambda), \gamma}^{A, \eta}(x-\gamma(U+V)(x, \lambda)), \omega\right)\right. \\
& \left.\quad-T\left(R_{N(\cdot, \lambda), \gamma}^{A, \eta}(y-\gamma(U+V)(y, \lambda)), \omega\right)\right\} \|^{q} \\
& \leq\left\|R_{N(\cdot, \lambda), \gamma}^{A, \eta}(x-\gamma(U+V)(x, \lambda))-R_{N(\cdot, \lambda), \gamma}^{A, \eta}(y-\gamma(U+V)(y, \lambda))\right\|^{q} \\
& -q \rho\left\langle T\left(R_{N(\cdot, \lambda), \gamma}^{A, \eta}(x-\gamma(U+V)(x, \lambda)), \omega\right)\right. \\
& \quad-T\left(R_{N(\cdot, \lambda), \gamma}^{A, \eta}(y-\gamma(U+V)(y, \lambda)), \omega\right), J_{q}\left(R_{N(\cdot, \lambda), \gamma}^{A, \eta}(x-\gamma(U+V)(x, \lambda))\right. \\
& \left.\left.\quad-R_{N \cdot(\cdot, \lambda), \gamma}^{A,,}(y-\gamma(U+V)(y, \lambda))\right)\right\rangle \\
& +c_{q} \rho^{q} \| T\left(R_{N(\cdot, \lambda), \gamma}^{A, \eta}(x-\gamma(U+V)(x, \lambda)), \omega\right) \\
& \quad-T\left(R_{N(\cdot, \lambda), \gamma}^{A, \eta}(y-\gamma(U+V)(y, \lambda)), \omega\right) \|^{q} \\
& \leq\left(1-q \rho \delta+c_{q} \rho^{q} \lambda_{T}^{q}\right) \| R_{N(\cdot, \lambda), \gamma}^{A, \eta}(x-\gamma(U+V)(x, \lambda)) \\
& -R_{N(\cdot, \lambda), \gamma}^{A, \eta}(y-\gamma(U+V)(y, \lambda)) \|^{q} \\
& \leq\left(1-q \rho \delta+c_{q} \rho^{q} \lambda_{T}^{q}\right)\left(\frac{\tau^{q-1}}{r-\lambda m}\right)^{q}(\|x-y-\gamma(V(x, \lambda)-V(y, \lambda))\| \\
& +\gamma\|U(x, \lambda)-U(y, \lambda)\|)^{q} .
\end{aligned}
$$


Since $V$ is $\alpha$-strongly accretive and $\lambda_{V}$-Lipschitz continuous with respect to the first argument,

$$
\begin{aligned}
& \|x-y-\gamma(V(x, \lambda)-V(y, \lambda))\|^{q} \\
\leq & \|x-y\|^{q}-q \gamma\left\langle V(x, \lambda)-V(y, \lambda), J_{q}(x-y)\right\rangle \\
& +c_{q} \gamma^{q}\|V(x, \lambda)-V(y, \lambda)\|^{q} \\
\leq & \left(1-\alpha q \gamma+c_{q} \gamma^{q} \lambda_{V}^{q}\right)\|x-y\|^{q} .
\end{aligned}
$$

By the $\lambda_{U}$-Lipschitz continuity of $U$, we have

$$
\|U(x, \lambda)-U(y, \lambda)\| \leq \lambda_{U}\|x-y\| .
$$

By the $\lambda_{S}$-Lipschitz continuity of $S,(3.5)$ and (3.6), we obtain

$$
\begin{aligned}
& \left\|S\left(R_{N(\cdot, \lambda), \gamma}^{A, \eta}(x-\gamma(U+V)(x, \lambda)), \omega\right)-S\left(R_{N(\cdot, \lambda), \gamma}^{A, \eta}(y-\gamma(U+V)(y, \lambda)), \omega\right)\right\| \\
\leq & \left.\left.\lambda_{S} \| R_{N(\cdot, \lambda), \gamma}^{A, \eta}(x-\gamma(U+V)(x, \lambda)), \omega\right)-R_{N(\cdot, \lambda), \gamma}^{A, \eta}(y-\gamma(U+V)(y, \lambda)), \omega\right) \| \\
\leq & \lambda_{S} \frac{\tau^{q-1}}{r-\lambda m}(\|x-y-\gamma(V(x, \lambda)-V(y, \lambda))\|+\gamma\|U(x, \lambda)-U(y, \lambda)\|) \\
\leq & \frac{\lambda_{S} \tau^{q-1}}{r-\lambda m}\left[\left(1-\alpha q \gamma+c_{q} \gamma^{q} \lambda_{V}^{q}\right)^{\frac{1}{q}}+\gamma \lambda_{U}\right]\|x-y\| .
\end{aligned}
$$

By (3.3)-(3.7), we have

$$
\begin{aligned}
& \|F(x, \omega, \lambda)-F(y, \omega, \lambda)\| \\
\leq & \left(\frac{\tau^{q-1}}{r-\rho m}\right)\left(\frac{\tau^{q-1}}{r-\lambda m}\right)\left[\left(1-\alpha q \gamma+c_{q} \gamma^{q} \lambda_{V}^{q}\right)^{\frac{1}{q}}+\gamma \lambda_{U}\right] \\
& \quad\left[\left(1-q \rho \delta+c_{q} \rho^{q} \lambda_{T}^{q}\right)^{\frac{1}{q}}+\rho \lambda_{S}\right]\|x-y\| \\
= & \left(\frac{\tau^{q-1}}{r-\rho m}\right)\left(\frac{\tau^{q-1}}{r-\lambda m}\right) \theta_{1} \theta_{2}\|x-y\| \\
= & \theta\|x-y\|,
\end{aligned}
$$

where $\theta_{1}=\left(1-\alpha q \gamma+c_{q} \gamma^{q} \lambda_{V}^{q}\right)^{\frac{1}{q}}+\gamma \lambda_{U}, \theta_{2}=\left(1-q \rho \delta+c_{q} \rho^{q} \lambda_{T}^{q}\right)^{\frac{1}{q}}+\rho \lambda_{S}$ and $\theta=\left(\frac{\tau^{q-1}}{r-\rho m}\right)\left(\frac{\tau^{q-1}}{r-\lambda m}\right) \theta_{1} \theta_{2}$. It follows from condition (3.2) that $\theta<1$. Thus, (3.8) implies that $F$ is a contractive mapping which is uniform with respect to $(\omega, \lambda) \in \Omega \times \Lambda$.

(2) Since $F(x, \omega, \lambda)$ is a uniform $\theta$-contractive mapping with respect to $(\omega, \lambda) \in \Omega \times \Lambda$, by the Banach fixed point theorem, $F(x, \omega, \lambda)$ has a fixed point $\bar{x}(\omega, \lambda)$ for each $(\omega, \lambda) \in \Omega \times \Lambda$. By Lemma 3.1, $S(\omega, \lambda) \neq \phi$. For each $(\omega, \lambda) \in \Omega \times \Lambda$, let $\left(x_{n}, y_{n}\right) \in S(\omega, \lambda)$ and $x_{n} \rightarrow x_{0}, y_{n} \rightarrow y_{0}$ as $n \rightarrow \infty$. Then we have

$$
x_{n} \in F\left(x_{n}, \omega, \lambda\right), \quad n=1,2, \ldots
$$


By (1), we have

$$
\left\|F\left(x_{n}, \omega, \lambda\right)-F(x, \omega, \lambda)\right\| \leq \theta\left\|x_{n}-x\right\|
$$

It follows that

$$
\begin{aligned}
\left\|x_{0}-F\left(x_{0}, \omega, \lambda\right)\right\| \leq & \left\|x_{0}-x_{n}\right\|+\left\|x_{n}-F\left(x_{n}, \omega, \lambda\right)\right\| \\
& +\left\|F\left(x_{n}, \omega, \lambda\right)-F\left(x_{0}, \omega, \lambda\right)\right\| \\
\leq & (1+\theta)\left\|x_{n}-x_{0}\right\| \\
& \rightarrow 0 \quad \text { as } n \rightarrow \infty .
\end{aligned}
$$

Hence we have $x_{0} \in F\left(x_{0}, \omega, \lambda\right)$. From Lemma 3.1 we have $\left(x_{0}, y_{0}\right) \in S(\omega, \lambda)$. Therefore $S(\omega, \lambda)$ is a nonempty closed subset of $E$.

Theorem 3.2. Under the hypotheses of Theorem 3.1, further assume that for any $x, y \in E$, the mappings $\omega \mapsto S(x, \omega), \omega \mapsto T(x, \omega), \lambda \mapsto U(y, \lambda)$ and $\lambda \mapsto V(y, \lambda)$ are Lipschitz continuous with constants $l_{S}, l_{T}, l_{U}, l_{V}$, respectively. Suppose that for any $(t, \omega \cdot \bar{\omega}) \in E \times \Omega \times \Omega$ and $(z, \lambda, \bar{\lambda}) \in E \times \Lambda \times \Lambda$,

$$
\begin{gathered}
\left\|R_{M(\cdot, \omega), \rho}^{A, \eta}(t)-R_{M(\cdot, \bar{\omega}), \rho}^{A, \eta}(t)\right\| \leq \mu\|\omega-\bar{\omega}\|, \\
\left\|R_{N(\cdot, \lambda), \gamma}^{A, \eta}(z)-R_{M(\cdot, \bar{\lambda}), \gamma}^{A, \eta}(z)\right\| \leq \tau\|\lambda-\bar{\lambda}\|,
\end{gathered}
$$

where $\mu>0$ and $\tau>0$ are two constants.

Then the solution $(x(\omega, \lambda), y(\omega, \lambda))$ for the system of parametric generalized nonlinear mixed quasi-variational inclusions with $(A, \eta)$-accretive mappings in q-uniformly smooth Banach spaces (2.1) is Lipschitz continuous.

Proof. For each $(\omega, \lambda),(\bar{\omega}, \bar{\lambda}) \in \Omega \times \Lambda$, by Theorem 3.1, $S(\omega, \lambda)$ and $(S(\bar{\omega}, \bar{\lambda})$ are both nonempty closed subsets. Also, $F(x, \omega, \lambda)$ and $F(x, \bar{\omega}, \bar{\lambda})$ are contractive mappings with same constant $\theta \in(0,1)$ and have fixed points $x(\omega, \lambda)$ and $x(\bar{\omega}, \bar{\lambda})$, respectively. For any fixed $(\omega, \lambda),(\bar{\omega}, \bar{\lambda}) \in \Omega \times \Lambda$, we have

$$
\begin{aligned}
& \|x(\omega, \lambda)-x(\bar{\omega}, \bar{\lambda})\| \\
= & \|F(x(\omega, \lambda), \omega, \lambda)-F(x(\bar{\omega}, \bar{\lambda}), \bar{\omega}, \bar{\lambda})\| \\
\leq & \|F(x(\omega, \lambda), \omega, \lambda)-F(x(\bar{\omega}, \bar{\lambda}), \omega, \lambda)\| \\
& \quad+\|F(x(\bar{\omega}, \bar{\lambda}), \omega, \lambda)-F(x(\bar{\omega}, \bar{\lambda}), \bar{\omega}, \bar{\lambda})\| \\
\leq & \theta\|x(\omega, \lambda)-x(\bar{\omega}, \bar{\lambda})\|+\|F(x(\bar{\omega}, \bar{\lambda}), \omega, \lambda)-F(x(\bar{\omega}, \bar{\lambda}), \bar{\omega}, \bar{\lambda})\|,
\end{aligned}
$$

which implies that

$$
\|x(\omega, \lambda)-x(\bar{\omega}, \bar{\lambda})\| \leq \frac{1}{1-\theta}\|F(x(\bar{\omega}, \bar{\lambda}), \omega, \lambda)-F(x(\bar{\omega}, \bar{\lambda}), \bar{\omega}, \bar{\lambda})\| .
$$

By condition (3.9), we have

$$
\begin{aligned}
& \|F(x(\bar{\omega}, \bar{\lambda}), \omega, \lambda)-F(x(\bar{\omega}, \bar{\lambda}), \bar{\omega}, \bar{\lambda})\| \\
\leq & \| R_{M(\cdot, \omega), \rho}^{A, \eta}\left[R_{N(\cdot, \lambda), \gamma}^{A, \eta}(x(\bar{\omega}, \bar{\lambda})-\gamma(U+V)(x(\bar{\omega}, \bar{\lambda}), \lambda))\right.
\end{aligned}
$$




$$
\begin{aligned}
& \left.-\rho(S+T)\left(R_{N(\cdot, \lambda), \gamma}^{A, \eta}(x(\bar{\omega}, \bar{\lambda})-\gamma(U+V)(x(\bar{\omega}, \bar{\lambda}), \lambda)), \omega\right)\right] \\
& -R_{M(\cdot, \omega), \rho}^{A, \eta}\left[R_{N(\cdot, \bar{\lambda}), \gamma}^{A, \eta}(x(\bar{\omega}, \bar{\lambda})-\gamma(U+V)(x(\bar{\omega}, \bar{\lambda}), \bar{\lambda}))\right. \\
& \left.-\rho(S+T)\left(R_{N(\cdot, \bar{\lambda}), \gamma}^{A, \eta}(x(\bar{\omega}, \bar{\lambda})-\gamma(U+V)(x(\bar{\omega}, \bar{\lambda}), \bar{\lambda})), \bar{\omega}\right)\right] \| \\
& +\| R_{M(\cdot, \omega), \rho}^{A, \eta}\left[R_{N(\cdot, \bar{\lambda}), \gamma}^{A, \eta}(x(\bar{\omega}, \bar{\lambda})-\gamma(U+V)(x(\bar{\omega}, \bar{\lambda}), \bar{\lambda})),\right. \\
& \left.-\rho(S+T)\left(R_{N(\cdot, \bar{\lambda}), \gamma}^{A, \eta}(x(\bar{\omega}, \bar{\lambda})-\gamma(U+V)(x(\bar{\omega}, \bar{\lambda}), \bar{\lambda})), \bar{\omega}\right)\right] \\
& -R_{M(\cdot, \bar{\omega}), \rho}^{A, \eta}\left[R_{N(\cdot, \bar{\lambda}), \gamma}^{A, \eta}(x(\bar{\omega}, \bar{\lambda})-\gamma(U+V)(x(\bar{\omega}, \bar{\lambda}), \bar{\lambda}))\right. \\
& \left.-\rho(S+T)\left(R_{N(\cdot, \bar{\lambda}), \gamma}^{A, \eta}(x(\bar{\omega}, \bar{\gamma})-\gamma(U+V)(x(\bar{\omega}, \bar{\lambda}), \bar{\lambda})), \bar{\omega}\right)\right] \| \\
& \leq \frac{\tau^{q-1}}{r-\rho m}\left[\| R_{N(\cdot, \lambda), \gamma}^{A, \eta}(x(\bar{\omega}, \bar{\lambda})-\gamma(U+V)(x(\bar{\omega}, \bar{\lambda}), \lambda))\right. \\
& -R_{N(\cdot, \bar{\lambda}), \gamma}^{A, \eta}(x(\bar{\omega}, \bar{\lambda})-\gamma(U+V)(x(\bar{\omega}, \bar{\lambda}), \bar{\lambda})) \\
& -\rho\left\{T\left(R_{N(\cdot, \lambda), \gamma}^{A, \eta}(x(\bar{\omega}, \bar{\lambda})-\gamma(U+V)(x(\bar{\omega}, \bar{\lambda}), \lambda)), \omega\right)\right. \\
& \left.-T\left(R_{N(\cdot, \bar{\lambda}), \gamma}^{A, \eta}(x(\bar{\omega}, \bar{\lambda})-\gamma(U+V)(x(\bar{\omega}, \bar{\lambda}), \bar{\lambda})), \bar{\omega}\right)\right\} \| \\
& +\rho \| S\left(R_{N(\cdot, \lambda), \gamma}^{A, \eta}(x(\bar{\omega}, \bar{\lambda})-\gamma(U+V)(x(\bar{\omega}, \bar{\lambda}), \lambda)), \omega\right) \\
& \left.-S\left(R_{N(\cdot, \bar{\lambda}), \gamma}^{A, \eta}(x(\bar{\omega}, \bar{\lambda})-\gamma(U+V)(x(\bar{\omega}, \bar{\lambda}), \bar{\lambda})), \bar{\omega}\right) \|\right] \\
& +\mu\|\omega-\bar{\omega}\| \\
& \leq \frac{\tau^{q-1}}{r-\rho m}\left[\| R_{N(\cdot, \lambda), \gamma}^{A, \eta}(x(\bar{\omega}, \bar{\lambda})-\gamma(U+V)(x(\bar{\omega}, \bar{\lambda}), \lambda))\right. \\
& -R_{N(\cdot, \lambda), \gamma}^{A, \eta}(x(\bar{\omega}, \bar{\lambda})-\gamma(U+V)(x(\bar{\omega}, \bar{\lambda}), \bar{\lambda})) \\
& -\rho\left\{T\left(R_{N(\cdot, \lambda), \gamma}^{A, \eta}(x(\bar{\omega}, \bar{\lambda})-\gamma(U+V)(x(\bar{\omega}, \bar{\lambda}), \lambda)), \omega\right)\right. \\
& \left.-T\left(R_{N(\cdot, \lambda), \gamma}^{A, \eta}(x(\bar{\omega}, \bar{\lambda})-\gamma(U+V)(x(\bar{\omega}, \bar{\lambda}), \bar{\lambda})), \omega\right)\right\} \| \\
& +\| R_{N(\cdot, \lambda), \gamma}^{A, \eta}(x(\bar{\omega}, \bar{\lambda})-\gamma(U+V)(x(\bar{\omega}, \bar{\lambda}), \bar{\lambda})) \\
& -R\left(_{N(\cdot, \bar{\lambda}), \gamma}(x(\bar{\omega}, \bar{\lambda})-\gamma(U+V)(x(\bar{\omega}, \bar{\lambda}), \bar{\lambda})) \|\right. \\
& +\rho\left\|T\left(R_{N(\cdot, \lambda), \gamma}^{A, \eta}(x(\bar{\omega}, \bar{\lambda})-\gamma(U+V)(x(\bar{\omega}, \bar{\lambda}), \bar{\lambda})), \omega\right)\right\| \\
& -T\left(R_{N(\cdot, \lambda), \gamma}^{A, \eta}(x(\bar{\omega}, \bar{\lambda})-\gamma(U+V)(x(\bar{\omega}, \bar{\lambda}), \bar{\lambda})), \bar{\omega}\right) \| \\
& +\rho \| T\left(R_{N(\cdot, \lambda), \gamma}^{A, \eta}(x(\bar{\omega}, \bar{\lambda})-\gamma(U+V)(x(\bar{\omega}, \bar{\lambda}), \bar{\lambda})), \bar{\omega}\right) \\
& -T\left(R_{N(\cdot, \bar{\lambda}), \gamma}^{A, \eta}(x(\bar{\omega}, \bar{\lambda})-\gamma(U+V)(x(\bar{\omega}, \bar{\lambda}), \bar{\lambda})), \bar{\omega}\right) \| \\
& +\rho \| S\left(R_{N(\cdot, \lambda), \gamma}^{A, \eta}(x(\bar{\omega}, \bar{\lambda})-\gamma(U+V)(x(\bar{\omega}, \bar{\lambda}), \lambda)), \omega\right) \\
& -S\left(R_{N(\cdot, \lambda), \gamma}^{A, \eta}(x(\bar{\omega}, \bar{\lambda})-\gamma(U+V)(x(\bar{\omega}, \bar{\lambda}), \bar{\lambda})), \bar{\omega}\right) \| \\
& +\rho\left\|S\left(R_{N(\cdot, \lambda), \gamma}^{A, \eta}(x(\bar{\omega}, \bar{\lambda})-\gamma(U+V)(x(\bar{\omega}, \bar{\lambda}), \bar{\lambda})), \bar{\omega}\right)\right\|
\end{aligned}
$$




$$
\begin{aligned}
& -S\left(R_{N(\cdot, \bar{\lambda}), \gamma}^{A, \eta}(x(\bar{\omega}, \bar{\lambda})-\gamma(U+V)(x(\bar{\omega}, \bar{\lambda}), \bar{\lambda})), \bar{\omega}\right) \| \\
+ & \mu\|\omega-\bar{\omega}\| .
\end{aligned}
$$

The $\delta$-strongly accretivity of $T$ and the Lipschitz continuity of $T, U$ and $V$ imply

$$
\begin{aligned}
& \| R_{N(\cdot, \lambda), \gamma}^{A, \eta}(x(\bar{\omega}, \bar{\lambda})-\gamma(U+V)(x(\bar{\omega}, \bar{\lambda}), \lambda)) \\
& -R_{N(\cdot, \lambda), \gamma}^{A, \eta}(x(\bar{\omega}, \bar{\lambda})-\gamma(U+V)(x(\bar{\omega}, \bar{\lambda}), \bar{\lambda})) \\
& -\rho\left\{T\left(R_{N(\cdot, \lambda), \gamma}^{A, \eta}(x(\bar{\omega}, \bar{\lambda})-\gamma(U+V)(x(\bar{\omega}, \bar{\lambda}), \lambda)), \omega\right)\right. \\
& \left.-T\left(R_{N(\cdot, \lambda), \gamma}^{A, \eta}(x(\bar{\omega}, \bar{\lambda})-\gamma(U+V)(x(\bar{\omega}, \bar{\lambda}), \bar{\lambda})), \omega\right)\right\} \|^{q} \\
& \leq \| R_{N(\cdot, \lambda), \gamma}^{A, \eta}(x(\bar{\omega}, \bar{\lambda})-\gamma(U+V)(x(\bar{\omega}, \bar{\lambda}), \lambda)) \\
& -R_{N(\cdot, \lambda), \gamma}^{A, \eta}(x(\bar{\omega}, \bar{\lambda})-\gamma(U+V)(x(\bar{\omega}, \bar{\lambda}), \bar{\lambda})) \|^{q} \\
& -q \rho\left\langle T\left(R_{N(\cdot, \lambda), \gamma}^{A, \eta}(x(\bar{\omega}, \bar{\lambda})-\gamma(U+V)(x(\bar{\omega}, \bar{\lambda}), \lambda)), \omega\right)\right. \\
& -T\left(R_{N(\cdot, \lambda), \gamma}^{A, \eta}(x(\bar{\omega}, \bar{\lambda})-\gamma(U+V)(x(\bar{\omega}, \bar{\lambda}), \bar{\lambda})), \omega\right), \\
& J_{q}\left(R_{N(\cdot, \lambda), \gamma}^{A, \eta}(x(\bar{\omega}, \bar{\lambda})-\gamma(U+V)(x(\bar{\omega}, \bar{\lambda}), \lambda))\right. \\
& \left.\left.-R_{N(\cdot, \lambda), \gamma}^{A, \eta}(x(\bar{\omega}, \bar{\lambda})-\gamma(U+V)(x(\bar{\omega}, \bar{\lambda}), \bar{\lambda}))\right)\right\rangle \\
& +c_{q} \rho^{q} \| T\left(R_{N(\cdot, \lambda), \gamma}^{A, \eta}(x(\bar{\omega}, \bar{\lambda})-\gamma(C+D)(x(\bar{\omega}, \bar{\lambda}), \lambda)), \omega\right) \\
& -T\left(R_{N(\cdot, \lambda), \gamma}^{A, \eta}(x(\bar{\omega}, \bar{\lambda})-\gamma(C+D)(x(\bar{\omega}, \bar{\lambda}), \bar{\lambda})), \omega\right) \|^{q} \\
& \leq\left(1-q \rho \delta+c_{q} \rho^{q} \lambda_{T}^{q}\right) \| R_{N(\cdot, \lambda), \gamma}^{A, \eta}(x(\bar{\omega}, \bar{\lambda})-\gamma(U+V)(x(\bar{\omega}, \bar{\lambda}), \lambda)) \\
& -R_{N(\cdot, \lambda), \gamma}^{A, \eta}(x(\bar{\omega}, \bar{\lambda})-\gamma(U+V)(x(\bar{\omega}, \bar{\lambda}), \bar{\lambda})) \|^{q} \\
& \leq\left(1-q \rho \delta+c_{q} \rho^{q} \lambda_{T}^{q}\right) \frac{\tau^{q(q-1)} \gamma^{q}}{(r-\lambda m)^{q}}(\|U(x(\bar{\omega}, \bar{\lambda}), \lambda)-U(x(\bar{\omega}, \bar{\lambda}) \bar{\lambda})\| \\
& +\|V(x(\bar{\omega}, \bar{\lambda}), \lambda)-V(x(\bar{\omega}, \bar{\lambda}), \bar{\lambda})\|)^{q} \\
& \leq\left(1-q \rho \delta+c_{q} \rho^{q} \lambda_{T}^{q}\right) \frac{\tau^{q(q-1)} \gamma^{q}}{(r-\lambda m)^{q}}\left(l_{U}+l_{V}\right)^{q}\|\lambda-\bar{\lambda}\|^{q} .
\end{aligned}
$$

By condition (3.9), we have

$$
\begin{aligned}
& \quad \| R_{N(\cdot, \lambda), \gamma}^{A, \eta}(x(\bar{\omega}, \bar{\lambda})-\gamma(U+V)(x(\bar{\omega}, \bar{\lambda}), \bar{\lambda})) \\
& \quad-R_{N(\cdot, \bar{\lambda}), \gamma}^{A, \eta}(x(\bar{\omega}, \bar{\lambda})-\gamma(U+V)(x(\bar{\omega}, \bar{\lambda}), \bar{\lambda})) \| \\
& \leq \tau\|\lambda-\bar{\lambda}\| .
\end{aligned}
$$

By the $l_{T}$-Lipschitz continuity of $T$, we have

$$
\begin{aligned}
& \| T\left(R_{N(\cdot, \lambda), \gamma}^{A, \eta}(x(\bar{\omega}, \bar{\lambda})-\gamma(U+V)(x(\bar{\omega}, \bar{\lambda}), \bar{\lambda}), \omega)\right. \\
& \quad-T\left(R_{N(\cdot, \lambda), \gamma}^{A, \eta}(x(\bar{\omega}, \bar{\lambda})-\gamma(U+V)(x(\bar{\omega}, \bar{\lambda}), \bar{\lambda})), \bar{\omega}\right) \|
\end{aligned}
$$




$$
\leq l_{T}\|\omega-\bar{\omega}\| .
$$

By using same argument, we can prove

$$
\begin{gathered}
\| T\left(R_{N(\cdot, \lambda), \gamma}^{A, \eta}(x(\bar{\omega}, \bar{\lambda})-\gamma(U+V)(x(\bar{\omega}, \bar{\lambda}), \bar{\lambda})), \bar{\omega}\right) \\
-T\left(R_{N(\cdot, \bar{\lambda}), \gamma}^{A, \eta}(x(\bar{\omega}, \bar{\lambda})-\gamma(U+V)(x(\bar{\omega}, \bar{\lambda}), \bar{\lambda})), \bar{\omega}\right) \| \\
\leq \lambda_{T} \tau\|\lambda-\bar{\lambda}\|, \\
\| S\left(R_{N(\cdot, \lambda), \gamma}^{A, \eta}(x(\bar{\omega}, \bar{\lambda})-\gamma(U+V)(x(\bar{\omega}, \bar{\lambda}), \lambda)), \omega\right) \\
\quad-S\left(R_{N(\cdot, \lambda), \gamma}^{A, \eta}(x(\bar{\omega}, \bar{\lambda})-\gamma(U+V)(x(\bar{\omega}, \bar{\lambda}), \bar{\lambda})), \bar{\omega}\right) \| \\
\leq \| S\left(R_{N(\cdot, \lambda), \gamma}^{A, \eta}(x(\bar{\omega}, \bar{\lambda})-\gamma(U+V)(x(\bar{\omega}, \bar{\lambda}), \lambda)), \omega\right) \\
\quad-\quad S\left(R_{N(\cdot, \lambda), \gamma}^{A, \eta}(x(\bar{\omega}, \bar{\lambda})-\gamma(U+V)(x(\bar{\omega}, \bar{\lambda}), \lambda)), \bar{\omega}\right) \| \\
+\| S\left(R_{N(\cdot, \lambda), \gamma}^{A, \eta}(x(\bar{\omega}, \bar{\lambda})-\gamma(U+V)(x(\bar{\omega}, \bar{\lambda}), \lambda)), \bar{\omega}\right) \\
\quad-S\left(R_{N(\cdot, \lambda), \gamma}^{A, \eta}(x(\bar{\omega}, \bar{\lambda})-\gamma(U+V)(x(\bar{\omega}, \bar{\lambda}), \bar{\lambda})), \bar{\omega}\right) \| \\
\leq l_{S}\|\omega-\bar{\omega}\|+\frac{\lambda_{S} \tau^{q-1} \gamma}{r-\lambda m}[\|U(x(\bar{\omega}, \bar{\lambda}), \lambda)-U(x(\bar{\omega}, \bar{\lambda}), \bar{\lambda})\| \\
+\|V(x(\bar{\omega}, \bar{\lambda}), \lambda)-V(x(\bar{\omega}, \bar{\lambda}), \bar{\lambda})\|] \\
\leq l_{S}\|\omega-\bar{\omega}\|+\frac{\lambda_{S} \tau^{q-1} \gamma}{r-\lambda m}\left(l_{U}+l_{V}\right)\|\lambda-\bar{\lambda}\|, \\
\| S\left(R_{N(\cdot, \lambda), \gamma}^{A, \eta}(x(\bar{\omega}, \bar{\lambda})-\gamma(U+V)(x(\bar{\omega}, \bar{\lambda}), \bar{\lambda})), \bar{\omega}\right) \\
\quad-\quad S\left(R_{N(\cdot, \bar{\lambda}), \gamma}^{A, \eta}(x(\bar{\omega}, \bar{\lambda})-\gamma(U+V)(x(\bar{\omega}, \bar{\lambda}), \bar{\lambda})), \bar{\omega}\right) \| \\
\leq \lambda_{S} \tau\|\lambda-\bar{\lambda}\| .
\end{gathered}
$$

It follows from (3.10)-(3.17) that

$$
\begin{aligned}
& \|x(\omega, \lambda)-x(\bar{\omega}, \bar{\lambda})\| \\
& \leq \frac{1}{1-\theta}\left[\frac { \tau ^ { q - 1 } } { r - \rho m } \left\{\left(1-q \rho \delta+c_{q} \rho^{q} \lambda_{T}^{q}\right)^{\frac{1}{q}} \frac{\tau^{q-1} \gamma}{r-\rho m}\left(l_{U}+l_{V}\right)\|\lambda-\bar{\lambda}\|\right.\right. \\
& \quad+\tau\|\lambda-\bar{\lambda}\|+\rho l_{T}\|\omega-\bar{\omega}\|+\rho \lambda_{T} \tau\|\lambda-\bar{\lambda}\|+\rho l_{S}\|\omega-\bar{\omega}\| \\
& \left.\left.\quad+\rho \lambda_{S} \frac{\tau^{q-1} \gamma}{r-\lambda m}\left(l_{u}+l_{V}\right)\|\lambda-\bar{\lambda}\|+\rho \lambda_{S} \tau\|\lambda-\bar{\lambda}\|\right\}+\mu\|\omega-\bar{\omega}\|\right] \\
& =\frac{1}{1-\theta}\left[\frac{\tau^{q-1} \rho}{r-\rho m}\left(l_{S}+l_{T}\right)+\mu\right]\|\omega-\bar{\omega}\| \\
& \quad+\left(\frac{1}{1-\theta}\right)\left(\frac{\tau^{q-1}}{r-\rho m}\right)\left[\frac{\tau^{q-1}}{r-\rho m}\left(1-q \rho \delta+c_{q} \rho^{q} \lambda_{T}^{q}\right)^{\frac{1}{q}} \gamma\left(l_{U}+l_{V}\right)\right. \\
& \left.+\tau+\rho \lambda_{T} \tau+\frac{\tau^{q-1}}{r-\lambda m} \rho \lambda_{S} \gamma\left(l_{U}+l_{V}\right)+\rho \lambda_{S} \tau\right]\|\lambda-\bar{\lambda}\|,
\end{aligned}
$$


where $\theta$ is the constant of (3.8). This proves that $x(\omega, \lambda)$ is Lipschitz continuous in $(\omega, \lambda) \in \Omega \times \Lambda$.

On the other hand,

$$
\begin{aligned}
& y(\omega, \lambda)=R_{N(\cdot, \lambda), \gamma}^{A, \eta}(x(\omega, \lambda)-\gamma(U+V)(x(\omega, \lambda), \lambda)), \\
& y(\bar{\omega}, \bar{\lambda})=R_{N(\cdot, \bar{\lambda}), \gamma}^{A, \eta}(x(\bar{\omega}, \bar{\lambda})-\gamma(U+V)(x(\bar{\omega}, \bar{\lambda}), \bar{\lambda})) .
\end{aligned}
$$

Hence, we have

$$
\begin{aligned}
& \|y(\omega, \lambda)-y(\bar{\omega}, \bar{\lambda})\| \\
& \leq \| R_{N(\cdot, \lambda), \gamma}^{A, \eta}(x(\omega, \lambda)-\gamma(U+V)(x(\omega, \lambda), \lambda)) \\
& \quad \quad-R_{N(\cdot, \lambda), \gamma}^{A, \eta}(x(\bar{\omega}, \bar{\lambda})-\gamma(U+V)(x(\bar{\omega}, \bar{\lambda}), \bar{\lambda})) \| \\
& \quad+\| R_{N(\cdot, \lambda), \gamma}^{A, \eta}(x(\bar{\omega}, \bar{\lambda})-\gamma(U+V)(x(\bar{\omega}, \bar{\lambda}), \bar{\lambda})) \\
& \quad-R_{N(\cdot, \bar{\lambda}), \gamma}^{A, \eta}(x(\bar{\omega}, \bar{\lambda})-\gamma(C+D)(x(\bar{\omega}, \bar{\lambda}), \bar{\lambda})) \| \\
& \leq \frac{\tau^{q-1}}{r-\lambda m}[\|x(\omega, \lambda)-x(\bar{\omega}, \bar{\lambda})-\gamma(V(x(\omega, \lambda), \lambda)-V(x(\bar{\omega}, \bar{\lambda}), \lambda))\| \\
& \quad+\gamma\|V(x(\bar{\omega}, \bar{\lambda}), \lambda)-V(x(\bar{\omega}, \bar{\lambda}), \bar{\lambda})\|+\gamma\|U(x(\omega, \lambda), \lambda)-U(x(\bar{\omega}, \bar{\lambda}), \lambda)\| \\
& \quad+\gamma\|U(x(\bar{\omega}, \bar{\lambda}), \lambda)-U(x(\bar{\omega}, \bar{\lambda}), \bar{\lambda})\|]+\tau\|\lambda-\bar{\lambda}\| \\
& \leq \frac{\tau^{q-1}}{r-\lambda m}\left[\left(1-\alpha q \gamma+c_{q} \gamma^{q} \lambda_{V}^{q}\right)^{\frac{1}{q}}\|x(\omega, \lambda)-x(\bar{\omega}, \bar{\lambda})\|+\gamma l_{V}\|\lambda-\bar{\lambda}\|\right. \\
& \left.\quad+\gamma \lambda_{U}\|x(\omega, \lambda)-x(\bar{\omega}, \bar{\lambda})\|+\gamma l_{U}\|\lambda-\bar{\lambda}\|\right]+\tau\|\lambda-\bar{\lambda}\| \\
& =\frac{\tau^{q-1}}{r-\lambda m}\left[\left(1-\alpha q \gamma+c_{q} \gamma^{q} \lambda_{V}^{q}\right)^{\frac{1}{q}}+\gamma \lambda_{U}\right]\|x(\omega, \lambda)-x(\bar{\omega}, \bar{\lambda})\| \\
& \quad+\left[\frac{\tau^{q-1}}{r-\lambda m} \gamma\left(l_{U}+l_{V}\right)+\tau\right]\|\lambda-\bar{\lambda}\| .
\end{aligned}
$$

It follows from the Lipschitz continuity of $x(\omega, \lambda)$ that $y(\omega, \lambda)$ is Lipschitz continuous. This completes the proof of Theorem 3.2.

\section{References}

[1] R. P. Agarwal, N. J. Huang, and M. Y. Tan, Sensitivity analysis for a new system of generalized nonlinear mixed quasi-variational inclusions, Appl. Math. Lett. 17 (2004), 345-352.

[2] S. Dafermos, Sensitivity analysis in variational inequalities, Math. Operat. Res. 13 (1988), 421-434.

[3] Y. P. Fang and N. J. Huang, H-monotone operator and resolvent operator technique for variational inclusions, Appl. Math. Comput. 145 (2003), 795-803.

[4] _ H-accretive operators and resolvent operator technique for solving variational inclusions in Banach spaces, Appl. Math. Lett. 17 (2004), 647-653.

[5] H. Y. Lan, Y. J. Cho, and R. U. Verma, On nonlinear relaxed cocoercive variational inclusions involving $(A, \eta)$-accretive mappings in Banach spaces, Comput. Math. Appl. 51 (2006), 1529-1538. 
[6] R. N. Mukherjee and H. L. Verma, Sensitivity analysis of generalized variational inequalities, J. Math. Anal. Appl. 167 (1992), 299-304.

[7] M. A. Noor, Generalized algorithm and sensitivity analysis for variational inequalities, J. Appl. Math. Stoch. Anal. 5 (1992), 29-42.

[8] Y. H. Pan, Sensitivity analysis for general quasi-variational inequalities, J. Sichuan Normal Univ. 19 (1996), 56-59.

[9] J. W. Peng, On a new system of generalized mixed quasi-variational-like inclusions with $(H, \eta)$-accretive operators in real q-uniformly smooth Banach spaces, Nonlinear Anal. 68 (2008), 981-993.

[10] J. W. Peng and D. L. Zhu, Three-step iterative algorithm for a system of set-valued variational inclusions with $(H, \eta)$-monotone operators, Nonlinear Anal. 68 (2008), 139153.

[11] R. U. Verma, A-monotonicity and applications to nonlinear variational inclusions, J. Appl. Math. Stoch. Anal. 17 (2004), no. 2, 193-195.

[12] H. K. Xu, Inequalities in Banach spaces with applications, Nonlinear Anal. 16 (1991), no. $12,1127-1138$

[13] N. D. Yen, Lipschitz continuity of solution of variational inequalities with a parametric polyhedral constraint, Math. Operat. Res. 20 (1995), 607-708.

[14] D. Zeidler, Nonlinear Functional Analysis and its Applications II: Monotone Operators, Springer-Verlag, Berlin, 1985.

JAE UG JEONG

Department of Mathematics

Dongeui UniVERSITY

PusAn 614-714, Korea

E-mail address: jujeong@deu.ac.kr

Soo HWAN Kim

Department of Mathematics

Dongeui University

PUSAN 614-714, Korea

E-mail address: sootopology@hanmail.net 